\title{
Quantization of four-dimensional Abelian gravity
}

\author{
Bogusław Broda. * Piotr Bronowskif Marcin Ostrowski f \\ Department of Theoretical Physics, University of Eódz Pomorska 149/153, 90-236 Eódz, Poland
}

(Dated: October 7, 2018)

\begin{abstract}
An abelian version of standard general relativity in the Cartan-Palatini gauge-like formulation in four dimensions has been introduced. Traditional canonical analysis utilizing similarities to the akin Husain-Kuchař $S U(2)$ version of gravity has been performed. The model has been next quantized in the canonical path-integral Faddeev-Popov formalism yielding abelian $B F$ theory.
\end{abstract}

PACS numbers: 04.50.+h, 04.60.-m, 11.15.-q

\section{Introduction}

Quantization of full four-dimensional gravity is a longstanding difficult problem of theoretical physics. There are a lot of approaches to this problem, but two of them, loop gravity and superstring theory seem to be the most promising. As is well-known the standard perturbative quantum-field-theory approach to quantization of four-dimensional gravity fails because of nonrenormalizability. Quite another preliminary approach to quantization program of gravity consists in investigation of other, similar and simpler models. For example, gravity in lower dimensions (in three [1], or in two [2]) or other non-gravitational generally covariant models like topological field theory (e.g. [3] ). Proceeding in this spirit, we aim to introduce and quantize a very naturally defined, generally covariant, gravitation-like model in four dimensions. We will call our model abelian gravity because, as it follows from its definition, it is a direct abelian analog of the standard general relativity in the CartanPalatini gauge-like formulation. Our model is $U(1)$ or abelian gravity in the same sense as the Husain-Kuchar model is $S U(2)$ gravity [4]. We have also noticed that our model is akin to the "toy theory" introduced by Okołów [5]. It appears that the abelian gravity has many interesting properties. It is, in a formal hamiltonian sense, similar to the Husain-Kuchař $S U(2)$ gravity and even, though in a more limited sense, to standard gravity. Because of abelianity it is, in principle, simpler than the $S U(2)$ model but the formal structure of constraints is more complex (the constraints are not independent). In spite of the fact that our model is abelian, it is non-linear and quite non-trivial. Actually, the abelian gravity is a topological theory. It appears that the abelian gravity can be explicitly quantized and thus solved. We shall perform the standard Faddeev-Popov quantization procedure obtaining the solution in a closed form.

In the first part of the paper (section \) we define our model and perform standard canonical analysis. Our

\footnotetext{
*bobroda@uni.lodz.pl

†bronowski@gmail.com

† ostrowsk@merlin.phys.uni.lodz.pl

$\S$ michalszanecki@wp.pl
}

derivation of the canonical form is analogous to the one proposed by Husain and Kuchař in the case of $S U(2)$ gravity [4]. Qualitatively, the corresponding expressions are simpler that in the $S U(2)$ case but because of the non-invertibility of the set of one-forms $e_{i}^{A}$ the situation is more complex and to maintain three-dimensional symmetry we are forced to introduce a collection of constraints which are not independent.

In the second part of our paper (section III) we perform quantization of the abelian gravity in the formalism of path integrals. To this end we use the most explicit and reliable canonical Faddeev-Popov approach. In our case the whole procedure consists of several steps. In the first step, we must throw out one of the dependent constraints. In the second one, we perform a change of variables and reexpress the path integral back in an almost covariant form. Up to that moment everything is gaugeindependent. Next, we impose the axial gauge-fixing condition for ordinary $U(1)$ gauge symmetry, and "static" (vanishing momenta) gauge condition for diffeomorphiclike symmetry. The final step corresponds to the original Faddeev-Popov trick consisting in exchange of gauges trading off the canonical gauge condition for the covariant one. Strictly speaking, in our case the trick is different because we do not know the explicit covariant form of the gauge (diffeomorphic-like) symmetry. Finally, we show that the abelian gravity is equivalent to abelian $B F$ model.

\section{CANONICAL ANALYSIS}

The action of the four-dimensional abelian gravity is defined by

$$
S\left[A_{\mu}, e_{\mu}^{A}\right]=\frac{1}{4} \int_{\mathcal{M}} \mathrm{d}^{4} x \epsilon^{\mu \nu \lambda \sigma} \epsilon_{A B} e_{\mu}^{A} e_{\nu}^{B} F_{\lambda \sigma},
$$

where the "frame" one-forms $e_{\mu}^{A}$ belong to the real twodimensional representation of $U(1)$, and $F_{\mu \nu}=\partial_{\mu} A_{\nu}-$ $\partial_{\nu} A_{\mu}$ is the $U(1)$ gauge curvature two-form. The Greek space-time indices run form 0 to 3, i.e. $\mu, \nu, \lambda, \sigma=$ $0,1,2,3$, the capital Latin internal indices run from 1 to 2 , i.e. $A, B=1,2$. The alternating symbol $\epsilon^{0123}=1$, and $\epsilon_{12}=1$.

We observe that symbolically the action (1) is of the 
form $\sim \int e \wedge e \wedge R$, which is characteristic for fourdimensional gravity in the Cartan-Palatini formalism, where the gauge group $S O(1,3)$ has been replaced by $U(1)$. In particular, the cosmological term $\sim \int e \wedge e \wedge e \wedge e$ vanishes identically for $U(1)$.

Denoting

$$
e_{\mu}^{1} \equiv B_{\mu}, \quad e_{\mu}^{2} \equiv C_{\mu}
$$

we can rewrite the action (11) more conveniently as

$$
\begin{aligned}
S\left[A_{\mu}, B_{\mu}, C_{\mu}\right] & =\frac{1}{2} \int_{\mathcal{M}} \mathrm{d}^{4} x \epsilon^{\mu \nu \lambda \sigma} B_{\mu} C_{\nu} F_{\lambda \sigma}= \\
& =\int_{\mathcal{M}=\mathbb{R} \times \Sigma} \mathrm{d}^{4} x \epsilon^{\mu \nu \lambda \sigma} B_{\mu} C_{\nu} \partial_{\lambda} A_{\sigma}
\end{aligned}
$$

Besides diffeomorphisms, there is a larger symmetry group than $S O(2) \cong U(1)$ present in the action. Namely, the full group $S L(2, \mathbb{R})$, acting locally according to formulas

$$
\begin{aligned}
& B_{\mu}^{\prime}=a B_{\mu}+b C_{\mu}, \\
& C_{\mu}^{\prime}=c B_{\mu}+d C_{\mu},
\end{aligned}
$$

where $\left(\begin{array}{l}a b \\ c d\end{array}\right) \in S L(2, \mathbb{R})$, leaves the action intact. Since only a generator of the subgroup $S O(2) \cong U(1)$ appears among constraints, we can ignore the larger symmetry group in our further canonical quantization procedure.

We can now recast the action in the canonical form as

$$
\int_{\mathbb{R}} \mathrm{d} t \int_{\Sigma} \mathrm{d}^{3} x\left(E^{k} \dot{A}_{k}+\frac{1}{2} \epsilon^{i j k} \epsilon_{A B} e_{0}^{A} e_{i}^{B} F_{j k}+A_{0} \partial_{k} E^{k}\right)
$$

where the momentum density

$$
E^{k} \equiv \frac{1}{2} \epsilon^{i j k} \epsilon_{A B} e_{i}^{A} e_{j}^{B} \equiv \epsilon^{i j k} B_{i} C_{j}
$$

or vectorially, simply $\vec{E}=\vec{B} \times \vec{C}$, and surface terms have been (temporarily) discarded. Finally,

$$
S\left[A_{i}, E^{i} ; N^{i}, N\right]=\int_{\mathbb{R}} \mathrm{d} t \int_{\Sigma} \mathrm{d}^{3} x\left(E^{i} \dot{A}_{i}-N^{i} \mathcal{C}_{i}-N \mathcal{C}\right),
$$

where $N, N^{i}$ are Lagrange multipliers and $\mathcal{C}, \mathcal{C}_{i}$ are constraints,

$$
\begin{gathered}
\mathcal{C} \equiv \partial_{i} E^{i}=0 \\
\mathcal{C}_{i} \equiv E^{j} F_{i j}=0 .
\end{gathered}
$$

Here, $\mathcal{C}$ is the ordinary abelian Gauss condition, whereas $\mathcal{C}_{i}$ are (non-independent) spatial diffeomorphism constraints. In contradistinction to general relativity there is no hamiltonian constraint, and in contradistinction to the $S U(2)$ Husain-Kuchař model the constraints are not independent. We could rewrite the diffeomorphism constraints (8b) in a vector form

$$
\mathcal{C}_{i} \equiv \epsilon_{i j k} E^{j} F^{k}=0, \quad \overrightarrow{\mathcal{C}} \equiv \vec{E} \times \vec{F}=0
$$

where the dual connection $F^{k} \equiv \frac{1}{2} \epsilon^{i j k} F_{i j}$. Obviously,

$$
E^{i} \mathcal{C}_{i}=0
$$

identically, and therefore there are only two independent constraints instead of three. In fact, three constraints would be not admissible because we would get wrongly, negative number of degrees of freedom, i.e. $3-(1+3)=$ -1 . The twin condition

$$
F^{i} \mathcal{C}_{i}=0
$$

does not further limit the number of constraints because it reduces to the previous one upon the constraint condition itself. Namely, the "vector" constraint $\overrightarrow{\mathcal{C}}=0$, says that the vectors $\vec{E}$ and $\vec{F}$ should be parallel, $\vec{E} \| \vec{F}$, see Eq. (9), i.e.

$$
\vec{E} \propto \vec{F}
$$

and thus Eq. (11) reduces to Eq. (10). In other words, Eq. (12) means that $\vec{E}=\alpha \vec{F}$, and consequently only one component of $\vec{E}$ is arbitrary, i.e. $\alpha$. Since, instead of six components (three for each of the two vectors $\vec{E}$ and $\vec{F}$ ) we have only four, three for $\vec{F}$ and one for $\alpha$ corresponding to the single degree of freedom of $\vec{E} \quad(6-2=4)$. Therefore, we have effectively exactly two independent constraints out of the three $\mathcal{C}_{i}$ present in the action (7).

Now, we should check whether the canonical form of the action (7) together with the constraints (8a) and (8b) really corresponds to the "semi-covariant" form (5). First of all, we observe that the first expected identification,

$$
N \equiv-A_{0},
$$

directly follows from Eq. (5). Whereas the "vector" Lagrange multiplier is given by

$$
N^{i}=e_{0}^{A} e_{A}^{i}
$$

where a new auxiliary field $e_{A}^{i}$, the "semi-inverse" of $e_{i}^{A}$, has been introduced in Appendix. The use of $e_{A}^{i}$ yields some redundancy, three non-independent constraints instead of two, but the result assumes more symmetric form.

One can easily check that, as usually, the constraints (8a) and (8b) are generators of gauge transformation. Defining

$$
\mathcal{C}_{\alpha} \equiv \int \mathrm{d}^{3} x \alpha(x) \mathcal{C}(x), \quad \mathcal{C}_{\omega} \equiv \int \mathrm{d}^{3} x \omega^{i}(x) \mathcal{C}_{i}(x),
$$

we verify that

$$
\begin{aligned}
\delta_{\alpha} A_{i} & \equiv\left\{\mathcal{C}_{\alpha}, A_{i}\right\}=\partial_{i} \alpha \\
\delta_{\alpha} E^{i} & \equiv\left\{\mathcal{C}_{\alpha}, E^{i}\right\}=0
\end{aligned}
$$


and

$$
\begin{aligned}
\delta_{\omega} A_{i} & \equiv\left\{\mathcal{C}_{\omega}, A_{i}\right\}=\omega^{j} F_{i j}, \\
\delta_{\omega} E^{i} & \equiv\left\{\mathcal{C}_{\omega}, E^{i}\right\}=\partial_{j}\left(\omega^{i} E^{j}-\omega^{j} E^{i}\right) .
\end{aligned}
$$

Eqs. (16) and (17) denote ordinary abelian $U(1)$ gauge transformations, whereas (18) and (19) yield diffeomorphisms modulo $U(1)$ gauge transformation and the Gauss condition, respectively. One could easily redefine constraints to have diffeomorphisms in a "pure" form (see [4]) but it is unnecessary for our further purposes.

\section{QUANTIZATION}

We will use the most intuitive, easy and reliable canonical Faddeev-Popov (path-integral) quantization procedure [6]. Since the original Faddeev-Popov procedure is best suited to the case with independent constraints [7], one should discard, in our case, one of them, $\mathcal{C}_{3}$, say. Intuitively, presence of a non-independent constraint would correspond to a doubled Dirac delta and thus to a trivial singularity in the path integral. Setting $N^{3}=0$, the path integral assumes the following explicit form

$$
\begin{aligned}
Z= & \int \exp \left[\frac { i } { \hbar } \int _ { \mathbb { R } \times \Sigma } \mathrm { d } ^ { 4 } x \left(E^{i} \dot{A}_{i}+\epsilon_{a b} N^{a} E^{b} F^{3}\right.\right. \\
& \left.\left.-\epsilon_{a b} N^{a} E^{3} F^{b}+A_{0} \partial_{i} E^{i}\right)\right] \times \\
& \times G\left(A_{i}, E^{i}\right) \mathcal{D} A_{\mu} \mathcal{D} E^{i} \mathcal{D} N^{a},
\end{aligned}
$$

where $G\left(A_{i}, E^{i}\right)$ denotes, as yet unspecified, gauge part (gauge conditions and Faddeev-Popov ghosts), and $a, b=1,2$. Now, we perform a minor change of variables,

$$
H_{a} \equiv \epsilon_{a b} N^{b} E^{3},
$$

and consequently

$$
\mathcal{D} H_{a}=\epsilon_{a b} E^{3} \mathcal{D} N^{b},
$$

where we can treat $E^{3}$ as a constant. Then

$$
\begin{aligned}
Z= & \int \exp \left[\frac { i } { \hbar } \int _ { \mathbb { R } \times \Sigma } \mathrm { d } ^ { 4 } x \left(E^{i} \dot{A}_{i}-\frac{H_{a} E^{a}}{E^{3}} F^{3}+H_{a} F^{a}\right.\right. \\
& \left.\left.+A_{0} \partial_{i} E^{i}\right)\right] G\left(A_{i}, E^{i}\right) \mathcal{D} A_{\mu} \mathcal{D} E^{i} \epsilon \frac{\mathcal{D} H_{a}}{\left(E^{3}\right)^{2}}
\end{aligned}
$$

where $\epsilon= \pm 1$ is a (non-essential) regularization dependent constant coming from the minus sign present in Eq. (21). Finally, we can reconstruct a (quasi) covariant form of $Z$ introducing the functional Dirac delta. Namely,

$$
\begin{aligned}
Z= & \int \exp \left[\frac{i}{\hbar} \int_{\mathbb{R} \times \Sigma} \mathrm{d}^{4} x\left(E^{i} F_{0 i}+H_{i} F^{i}\right)\right] \times \\
& \times \delta\left(E^{i} H_{i}\right) G\left(A_{i}, E^{i}\right) \mathcal{D} A_{\mu} \mathcal{D} E^{i} \epsilon \frac{\mathcal{D} H_{i}}{E^{3}}
\end{aligned}
$$

where the functional integration with respect to an auxiliary (new) variable $H_{3}$ yields the Eq. (22) back. In
Eq. (23) the primarily discarded surface term has been recovered. Defining

$$
E^{i} \equiv \frac{1}{2} \epsilon^{0 i j k} \sigma_{j k}, \quad H_{i} \equiv \sigma_{0 i}
$$

we obtain explicit "covariantization" of the path integral in Eq. (23),

$$
Z=\int \exp \left(\frac{i}{\hbar} \int_{\mathcal{M}} \sigma \wedge F\right) \delta(\sigma \wedge \sigma) \frac{\epsilon G}{\sigma_{12}} \mathcal{D} A \mathcal{D} \sigma .
$$

Everything looks explicitly covariantly in Eq. (25) except possibly the fraction in the measure. It is interesting to note a similarity between Eq. (25) and the "toy model" presented in [5].

To proceed further we should chose convenient gauge conditions. We suggest the following canonical noncovariant gauge conditions

$$
\mathcal{G} \equiv A_{3}=0, \quad \mathcal{G}^{a} \equiv E^{a}=0,
$$

which we could call "axial-static" ones. The gauge conditions (26) fulfill the necessary equalities [6]

$$
\left\{\mathcal{G}, \mathcal{G}^{a}\right\}=\left\{\mathcal{G}^{a}, \mathcal{G}^{b}\right\}=0 .
$$

One can easily calculate the corresponding FaddeevPopov determinant

$$
\begin{gathered}
\operatorname{det} M \equiv\left|\begin{array}{cc}
\{\mathcal{C}, \mathcal{G}\} & \left\{\mathcal{C}, \mathcal{G}^{b}\right\} \\
\left\{\mathcal{C}_{a}, \mathcal{G}\right\} & \left\{\mathcal{C}_{a}, \mathcal{G}^{b}\right\}
\end{array}\right|=\left|\begin{array}{cc}
\partial_{3} & 0 \\
0 & \delta_{a}^{b} E^{3} \partial_{3}
\end{array}\right|= \\
=\left|\operatorname{det} \partial_{3}\right|^{3} \prod_{x}\left[E^{3}(x)\right]^{2} .
\end{gathered}
$$

Thus, the gauge-fixed form of the path integral (25) becomes

$$
\begin{aligned}
Z= & \int \exp \left(\frac{i}{\hbar} \int \sigma \wedge F\right) \delta(\sigma \wedge \sigma) \epsilon \sigma_{12}\left|\operatorname{det} \partial_{3}\right|^{3} \times \\
& \times \delta\left(A_{3}\right) \delta\left(E^{a}\right) \mathcal{D} A \mathcal{D} \sigma .
\end{aligned}
$$

Utilizing the gauge conditions for $E^{a}$, we transform Eq. (29) to

$$
\begin{gathered}
Z=\int \exp \left[\frac{i}{\hbar} \int \mathrm{d}^{4} x\left(E^{3} F_{03}+H_{i} F^{i}\right)\right] \times \\
\times \delta\left(E^{3} H_{3}\right) \epsilon E^{3}\left|\operatorname{det} \partial_{3}\right|^{3} \delta\left(A_{3}\right) \mathcal{D} A_{\mu} \mathcal{D} E^{3} \mathcal{D} H_{i},
\end{gathered}
$$

and finally, functionally integrating with respect to $\mathcal{D} H_{3}$ we obtain

$$
\begin{aligned}
Z= & \int \exp \left[\frac{i}{\hbar} \int \mathrm{d}^{4} x\left(E^{3} F_{03}+H_{a} F^{a}\right)\right] \times \\
& \times \epsilon\left|\operatorname{det} \partial_{3}\right|^{3} \delta\left(A_{3}\right) \mathcal{D} A_{\mu} \mathcal{D} E^{3} \mathcal{D} H_{a} .
\end{aligned}
$$

One could shorten the derivation of Eq. (31) from Eq. (20) but we insist on keeping just this longer derivation because of the excellent opportunity to present 
the intermediate (quasi) covariant form of $Z$ given by Eq. (25).

Now we should perform the procedure analogous to the Faddeev-Popov trick, consisting in replacing a noncovariant gauge by a covariant one and yielding an explicitly covariant form of the path integral. Actually, we do not invoke the original Faddeev-Popov trick because we do not have at our disposal a covariant version of (the "reduced" diffeomorphic) gauge transformations. This difficulty follows from the fact that the standard covariant form of diffeomorphic transformations is implemented by four generators corresponding to four constraints whereas we need only two of them. Instead, we will show that canonically quantized four-dimensional abelian $B F$ model assumes the form of Eq. (31). Let us recall the definition of the $B F$ theory [8]. The action is defined by

$$
S_{B F}\left[A_{\mu}, B_{\mu \nu}\right] \equiv \frac{1}{4} \int_{\mathcal{M}} \mathrm{d}^{4} x \epsilon^{\mu \nu \lambda \sigma} B_{\mu \nu} F_{\lambda \sigma}
$$

where $F_{\mu \nu}=\partial_{\mu} A_{\nu}-\partial_{\nu} A_{\mu}$ as earlier, and $B_{\mu \nu}$ is an abelian two-form.

Now,

$$
\begin{aligned}
S_{B F} & =\frac{1}{2} \int \epsilon^{\mu \nu \lambda \sigma} B_{\mu \nu} \partial_{\lambda} A_{\sigma}= \\
& =\int_{\mathbb{R}} \mathrm{d} t \int_{\Sigma} \mathrm{d}^{3} x\left(E^{i} \dot{A}_{i}+B_{0 i} F^{i}+A_{0} \partial_{i} E^{i}\right),
\end{aligned}
$$

where, this time, the momentum density

$$
E^{i} \equiv \frac{1}{2} \epsilon^{i j k} B_{j k}
$$

Then,

$$
S_{B F}\left[A_{i}, E^{i} ; N_{i}, N\right]=\int_{\mathbb{R} \times \Sigma} \mathrm{d}^{4} x\left(E^{i} \dot{A}_{i}-N_{i} \mathcal{C}^{i}-N \mathcal{C}\right),
$$

where, in this model, the Lagrange multipliers are simply given by

$$
N \equiv-A_{0}, \quad N_{i} \equiv B_{i 0}
$$

whereas the constraints

$$
\mathcal{C} \equiv \partial_{i} E^{i}=0, \quad \mathcal{C}^{i} \equiv F^{i}=0 .
$$

In this model the constraints $\mathcal{C}^{i}$ are not independent either because of the Bianchi identity

$$
\partial_{i} \mathcal{C}^{i} \equiv \partial_{i} F^{i}=0
$$

Setting $N_{3}=0$, as earlier, the path integral assumes the following form

$$
\begin{aligned}
Z= & \int \exp \left[\frac{i}{\hbar} \int_{\mathbb{R} \times \Sigma} \mathrm{d}^{4} x\left(E^{i} \dot{A}_{i}-N_{a} F^{a}+A_{0} \partial_{i} E^{i}\right)\right] \times \\
& \times G\left(A_{i}, E^{i}\right) \mathcal{D} A_{\mu} \mathcal{D} E^{i} \mathcal{D} N_{a}
\end{aligned}
$$

where $G\left(A_{i}, E^{i}\right)$ denotes an appropriate gauge term to be specified.

To proceed further we chose the gauge conditions of the form formally identical to (26). Obviously, Eqs. (27) are also fulfilled. The Faddeev-Popov determinant equals

$$
\operatorname{det} M \equiv\left|\begin{array}{cc}
\partial_{3} & 0 \\
0 & \epsilon_{a b} \partial_{3}
\end{array}\right|=\epsilon\left|\operatorname{det} \partial_{3}\right|^{3}
$$

where $\epsilon$ is the regularization dependent sign introduced earlier in our work. Now

$$
\begin{aligned}
Z= & \int \exp \left[\frac{i}{\hbar} \int \mathrm{d}^{4} x\left(E^{i} F_{0 i}-N_{a} F^{a}\right)\right] \times \\
& \times \epsilon\left|\operatorname{det} \partial_{3}\right|^{3} \delta\left(A_{3}\right) \delta\left(E^{a}\right) \mathcal{D} A_{\mu} \mathcal{D} E^{i} \mathcal{D} N_{a},
\end{aligned}
$$

which is equal to Eq. (31) if we set $H_{a} \equiv-N_{a}$, and apply the second functional Dirac delta with respect to $E^{a}$.

\section{CONCLUSIONS}

In the previous section we have managed to show that the abelian gravity and abelian $B F$ theory, upon an appropriate choice of non-covariant canonical gauge conditions in their canonical formulations, yield the same pathintegral formulas. Therefore, in spite of the fact that we do not know a covariant version of the diffeomorphiclike gauge transformations, we can use the $B F$ model to covariantly quantize the abelian gravity. The procedure of quantization of $B F$ models in covariant gauges is well-known [8]. It follows that the abelian gravity is a topological field theory, as it should, because of the simple count of the number of degrees of freedom, i.e. $3-[1+(3-1)]=0$. Although, from physical point of view, the abelian gravity does not resemble the standard gravity its formal canonical structure does. Therefore, we believe that the model of abelian gravity could be of use in theoretical physics applications.

Actually, the four-dimensional abelian gravity could be considered as a dual of four-dimensional abelian $B F$ model because it reproduces an equivalent system with other field content. Since in the abelian gravity the "local base" is spanned by the two vectors, $B_{\mu}$ and $C_{\mu}$, instead of four, one can interpret that model as a highly degenerated (topological) sector of the full (quantum) gravity. Therefore, one could speculate that what is known about the $B F$ system could be possibly used on gravitational side, but at the moment, we have not at our disposal any explicit example of this sort.

\section{Acknowledgments}

This work was supported in part by the Polish Ministry of Science and Higher Education Grant PBZ/MIN/008/P03/2003 and by the University of Eódź grant. 


\section{Appendix}

Let us define an auxiliary contravariant metric

$$
g^{A B} \equiv \delta^{i j} e_{i}^{A} e_{j}^{B}=\left(\begin{array}{cc}
B^{2} & B \cdot C \\
B \cdot C & C^{2}
\end{array}\right)
$$

Hence, the covariant metric $g_{A B}$, the (matrix) inverse of $g^{A B}$ is

$$
g_{A B}=\frac{1}{B^{2} C^{2}-(B \cdot C)^{2}}\left(\begin{array}{cc}
C^{2} & -B \cdot C \\
-B \cdot C & B^{2}
\end{array}\right) .
$$

Now, we define the "semi-inverse" $e_{A}^{i}$ of $e_{i}^{A}$, i.e.

$$
e_{A}^{i} \equiv \delta^{i j} g_{A B} e_{j}^{B}
$$

Actually,

$$
e_{i}^{A} e_{B}^{i}=\delta_{B}^{A}
$$

As an easy exercise, one can check that

$$
-N^{i} \mathcal{C}_{i}=\frac{1}{2} \epsilon^{i j k} \epsilon_{A B} e_{0}^{A} e_{i}^{B} F_{j k}
$$

where $N^{i}$ and $\mathcal{C}_{i}$ are defined by Eq. (14) and Eq. (8b), respectively.
[1] E. Witten, Nuclear Physics B 311, 46 (1988).

[2] D. Grumiller, W. Kummer, and D. Vassilevich, Physics Reports 369, 327 (2002).

[3] E. Witten, Communications in Mathematical Physics 117, 353 (1988).

[4] V. Husain and K. Kuchař, Physical Review D 42, 4070 (1990).
[5] A. Okołów, Arxiv preprint gr-qc/0605138 (2006).

[6] L. Faddeev and A. Slavnov, Gauge fields, introduction to quantum theory (Addison-Wesley, 1991).

[7] A. Slavnov and S. Frolov, Theoretical and Mathematical Physics 75, 470 (1988).

[8] M. Blau and G. Thompson, Ann. Phys 205, 130 (1991). 